\title{
Propriedades mecânicas e térmicas das blendas e nanocompósitos de UHMWPE/LLDPE/CNT para aplicação balística
}

\author{
Mechanical and thermal properties of \\ UHMWPE / LLDPE / CNT blends and \\ nanocomposites for ballistic \\ application
}

Carlos Alberto de Oliveira Couto ${ }^{1}$, Bruna Cristina da Silva ${ }^{1}$, Eduardo Henrique Backes ${ }^{2}$, Fábio Roberto Passador ${ }^{1}$

\footnotetext{
${ }^{1}$ Departamento de Ciência e Tecnologia, Universidade Federal de São Paulo, Rua Talim 330, CEP 12231-280, São José dos Campos, São Paulo, Brasil.

${ }^{2}$ Universidade Federal de São Carlos - Departamento de Engenharia de Materiais - Rodovia Washington Luís, Km 235, Caixa Postal 676, CEP 13565-905, São Carlos, São Paulo, Brasil.

e-mail: carlos.couto.sjc@gmail.com
}

\section{RESUMO}

O desenvolvimento de placas do nanocompósito de UHMWPE/LLDPE/CNT tem como objetivo a obtenção de um material para aplicação em blindagem balística híbrida de alto impacto em forma de multicamadas de cerâmica/polímero. O compósito polimérico é utilizado na base da blindagem com a função de absorver a energia cinética residual do projétil e dos estilhaços da cerâmica após o impacto. Para atender a estes requisitos o compósito polimérico precisa ter alta tenacidade à fratura, alta resistência mecânica e deformação controlada. Uma deformação exagerada da placa polimérica pode causar lesões aos usuários de coletes balísticos de proteção pessoal, por exemplo. Neste trabalho foi realizada a mistura de polietileno linear de baixa densidade (LLDPE) com polietileno de ultra alta massa molecular (UHMWPE) utilizando um homogeneizador de alta rotação com posterior prensagem a quente com a finalidade de melhorar a mistura dos componentes fundidos e facilitar o processo de fabricação das placas poliméricas. Para compensar as perdas de propriedades mecânicas das blendas em relação ao UHMWPE puro foram produzidos compósitos com adição de 0,$5 ; 1,0$ e 2,0 \% em massa de nanotubos de carbono funcionalizados com ácido nítrico. Os valores das propriedades mecânicas e térmicas dos compósitos foram comparados com as blendas poliméricas sem adição de nanotubos de carbono. As blendas poliméricas e nanocompósitos foram submetidos aos ensaios mecânicos de dureza Shore D e ensaio de tração uniaxial. Para avaliação da estabilidade térmica foram realizadas análises de calorimetria exploratória diferencial (DSC) e análise termogravimétrica (TGA), e para a avaliação morfológica dos nanocompósitos foi utilizado microscopia eletrônica de varredura (FEG-MEV).

Palavras-chave: Polietileno de ultra alta massa molecular, blendas poliméricas, nanotubos de carbono, nanocompósito, blindagem balística.

\section{ABSTRACT}

The purpose of developing UHMWPE/ LLDPE/ CNT nanocomposite plates is to obtain a material that can be applied in laminated ceramic/polymer structures for high-impact ballistic protection. The polymer nanocomposite is used in the backing plate of the armor structure to absorb the residual kinetic energy of the projectile and shards from the ceramic material after impact. The polymer nanocomposite must have high fracture toughness, high mechanical strength and controlled deformation, in order to comply with these requirements. For instance, extreme deformations of the backing plate may cause injury to users of ballistic protective vests. In this work, a blend of linear low-density polyethylene (LLDPE) and ultra-high molecular weight polyethylene (UHMWPE) was prepared, using a high-rotation homogenizer with subsequent hot pressing, in order to facilitate the manufacturing process of the polymer nanocomposite plates. Furthermore, carbon nanotubes $(0.5,1.0$ and $2.0 \mathrm{wt} \%)$, functionalized with nitric acid, were added to the polymer blend, in order 
to improve the mechanical properties of the resulting material. The mechanical and thermal properties of the polymer blend and the polymer nanocomposite were compared with each other. The polymer blends and polymer nanocomposites were submitted to mechanical tests such as Shore D hardness and uniaxial tensile tests. Differential scanning calorimetry (DSC) and thermogravimetric analysis (TGA) were performed to evaluate thermal stability of the various compositions. Finally, scanning electron microscopy (SEM-FEG) was performed to evaluate the morphology of the nanocomposites.

Keywords: Ultra high molecular weight polyethylene, polymer blends, carbon nanotubes, nanocomposite, ballistic protection.

\section{INTRODUÇÃO}

A blindagem híbrida consiste na utilização de dois ou mais materiais formando um "sanduíche", com objetivo de utilizar as propriedades diferentes e complementares dos materiais. Comumente a blindagem híbrida é composta por um material rígido e de alta dureza e outro que é um material dúctil e de grande alongamento.

A camada que recebe o impacto inicial é normalmente uma placa cerâmica, de extrema dureza e alto módulo de elasticidade, capaz de fraturar a ponta do projétil e dissipar a maior parte de sua energia cinética [1]. A outra camada deve ser formada por um material dúctil, capaz de se deformar sem se romper, e deve ser capaz de absorver a energia residual dos fragmentos do projétil e da cerâmica estilhaçada, através da transformação de energia cinética em energia de deformação plástica. A segunda camada, também chamada de base além da absorção da energia residual do sistema, deve possuir resistência suficiente para suportar as cargas subsequentes ao impacto, não podendo falhar nestes estágios iniciais [1].

Para o projeto de proteção balística devem ser observados alguns aspectos, tais como: o nível balístico da proteção, os métodos e processos de fabricação da proteção e as propriedades dos materiais empregados. Outros detalhes que precisam ser observados são a resistência da blindagem após determinado número de impactos (análise estatística de impactos), condições ambientais como calor, frio, chuva, limitação de espaço, custo, peso e ergonomia da blindagem e danos colaterais após o impacto [2,3].

Um material muito utilizado em proteções balísticas pessoais é UHMWPE na forma de fibras. As fibras de UHMWPE foram desenvolvidas na década de 1980, normalmente são fabricadas pelo método de fiação gel ou fiação do fundido. A estrutura de cadeias especiais e a técnica de processamento dotam a fibra com excelentes propriedades físicas e químicas, tais como alta resistência e módulo de elasticidade, de baixa densidade, resistência à corrosão, resistência ao impacto, excelente resistência ao stress crack, excelente resistência ao impacto, baixo coeficiente de atrito e capacidade de auto lubrificação. Estas proteções são desenvolvidas na forma de mantas confeccionadas a partir de fibras comerciais como a "Spectra" da Honeywell e "Dyneema" da DSM [4].

O polietileno de ultra alta massa molecular é um polímero sintético composto de cadeias saturadas, lineares ou ramificadas, cuja massa molar média é igual ou superior a $2,5 \times 10^{6} \mathrm{~g} \cdot \mathrm{mol}^{-1}$ e por possuir cadeias muito longas, o número de entrelaçamentos entre cadeias é muito mais elevado em comparação a outros tipos de polietileno e a temperatura de fusão cristalina $(\mathrm{Tm})$ é pouco mais elevada e situa-se na faixa de $130{ }^{\circ} \mathrm{C}$ e $136^{\circ} \mathrm{C}[5]$.

Suas moléculas de cadeias longas, de fácil deslizamento e estruturas semicristalinas garantem a grande resistência ao desgaste deste polímero [6]. Além disso, o UHMWPE apresenta cadeias até 10 vezes maiores do que o polietileno de alta densidade (HDPE) e uma combinação de propriedades únicas, como alta resistência ao impacto e à abrasão e baixo coeficiente de atrito [7]. A excelente resistência ao impacto do UHMWPE está relacionada com a sua elevada ductilidade; possui ainda um grande alongamento na ruptura (muito superior a 100\%) e, como resultado, uma grande capacidade de absorver energia antes da ruptura, propriedade fundamental para aplicação balística [8]. Uma vez que o peso molecular elevado do UHMWPE exibe uma viscosidade muito elevada no fundido, não é possível processá-lo por meio de técnicas convencionais tais como injeção, moldagem ou extrusão. Técnicas de fabricação especiais, como moldagem por compressão, extrusão por pistão hidráulico (RAM) ou sinterização são utilizadas [9].

Uma alternativa para diminuir a viscosidade do fundido pode ser a adição de uma segunda fase, com mesma estrutura química e com baixa viscosidade do fundido, como o polietileno linear de baixa densidade (LLDPE). Os polietilenos lineares de baixa densidade têm um comportamento mecânico superior ao polietileno de baixa densidade (LDPE). Embora possuam densidades semelhantes, suas estruturas são bastante diferentes, pois os polietilenos lineares de baixa densidade apresentam ramificações curtas ao longo de sua cadeia principal, diferentemente dos polietilenos de baixa densidade [10]. As ramificações de cadeias curtas têm influência sobre a morfologia e algumas propriedades físicas tais como rigidez, densidade, dureza e resistên- 
cia a tração, uma vez que a estrutura ramificada de algumas regiões das moléculas impede o arranjo perfeitamente ordenado das cadeias $[11,12]$.

Blendas poliméricas são sistemas poliméricos originários da mistura física de dois ou mais polímeros e/ou copolímeros, sem que haja um elevado grau de reações químicas entre eles. Para ser considerada uma blenda, os compostos devem ter concentração acima de $2 \%$ em massa do segundo componente [11]. As blendas poliméricas são comercialmente viáveis com propriedades específicas a cada aplicação e têm se mostrado uma excelente alternativa possibilitando a obtenção de materiais poliméricos para uma ampla gama de aplicações, com boa relação custo/desempenho [13].

O uso de UHMWPE tem sido limitado devido ao seu baixo módulo de Young e baixa tensão de ruptura, quando processado pelos processos convencionais, como moldagem por compressão e RAM para obtenção de placas. Neste contexto, muita atenção tem sido dada no desenvolvimento de compósitos de UHMWPE, a fim de melhorar as suas propriedades mecânicas e tribológicas [14].

Os nanotubos de carbono (CNT) têm se apresentado como promissores materiais para melhorar as propriedades de polímeros devido às suas propriedades excepcionais; alta resistência mecânica, alto módulo elástico, além das propriedades eletrônicas, entre outras [15]. Os CNT por possuírem grande área superficial e razão de aspecto são muito propensos a se aglomerarem, por esta razão, vários métodos para o desenvolvimento de nanocompósitos poliméricos tem sido propostos, incluindo diferentes técnicas de processamento e métodos de funcionalização das nanocargas afim de melhorar a dispersão e aumentar a adesão interfacial do reforço com a matriz polimérica e por conseguinte obter materiais com elevada resistência mecânica e ao desgaste [16-18].

Nesse trabalho foram desenvolvidas blendas poliméricas de UHMWPE/LLDPE com diferentes teores de LLDPE e nanocompósitos de UHMWPE/LLDPE/CNT com diferentes teores de CNT por mistura mecânica do fundido utilizando uma rota diferente de processamento através de homogeneizador de alta rotação e as propriedades térmicas, mecânicas e morfológicas foram avaliadas e correlacionadas buscando-se aplicação como material para blindagem balística.

\section{MATERIAIS E MÉTODOS}

Para o desenvolvimento desse trabalho foram utilizados:

- Polietileno de ultra alta massa molecular (UHMWPE), resina de especificação UTEC 6540 produzido pela Braskem com densidade de 0,925 g.cm ${ }^{-3}$ e viscosidade intrínseca de $28 \mathrm{dL} . \mathrm{g}^{-1}$ (ASTM D 4020).

- Polietileno linear de baixa densidade (LLDPE), resina de especificação IC 32, fornecida pela Braskem. de 29 g. 10

Essa resina é um copolímero de buteno, produzida pelo processo de solução, com índice de fluidez $\min ^{-1}\left(190{ }^{\circ} \mathrm{C} / 2,16 \mathrm{Kg}\right)$.

- Nanotubos de carbono de múltiplas camadas (CNT), fornecido pela Nanostructured and Amorphous Materials Inc. com 95\% de pureza, diâmetro interno menor que $8 \mathrm{~nm}$ e comprimento na ordem de $10-30 \mu \mathrm{m}$.

\subsection{Modificação superficial dos nanotubos de carbono}

As superfícies dos CNT foram modificadas com grupos carboxilas por reação de oxidação com ácido nítrico. Para a reação de oxidação, foram adicionados $4 \mathrm{~g}$ de CNT a $500 \mathrm{ml}$ de solução de $\mathrm{HNO}_{3}$ a 3 mol. $\mathrm{L}^{-1}$. Esta solução foi submetida a refluxo durante um período de 12 horas a $120{ }^{\circ} \mathrm{C}$. Após o período de oxidação, a solução foi centrifugada durante 20 minutos a $20^{\circ} \mathrm{C}$ e $4500 \mathrm{rpm}$, seguida de lavagem com água destilada e filtração por vácuo até a neutralização do pH da solução [19].

\subsection{Processamento das blendas e nanocompósitos poliméricos}

As blendas poliméricas de UHMWPE/LLDPE foram preparadas com dois teores de LLDPE (10 e $20 \%$ em massa) e os nanocompósitos poliméricos de UHMWPE/LLDPE/CNT foram preparados com 0,5; 1,0 e 2,0 \% em massa de CNT modificados utilizando um homogeneizador rotacional DRAIS, modelo $\mathrm{MH}-50 \mathrm{H}$ da $\mathrm{MH}$ Instrumentos com rotação de $3000 \mathrm{rpm}$. Nesse processo, a fusão/amolecimento dos componentes ocorreu por aquecimento viscoso decorrente do atrito. O tempo de mistura foi de aproximadamente 1 minuto. 
Após a mistura mecânica das blendas e nanocompósitos poliméricos, o material fundido/amolecido foi prensado a quente por um tempo médio de 4 minutos sob pressão de 7 bar a $180{ }^{\circ} \mathrm{C}$, utilizando uma prensa hidro-pneumática da $\mathrm{MH}$ Instrumentos, modelo PR8H. As amostras foram preparadas nas dimensões de 130 x $130 \times 3,2 \mathrm{~mm}^{3}$ e $170 \times 110 \times 3,2 \mathrm{~mm}^{3}$ para realização dos ensaios térmicos, mecânicos e estruturais. As placas mais longas foram cortadas em corpos de provas conforme norma ASTM D638-02a tipo I para os ensaios de tração, utilizando uma estampadeira da CEAST-Instron. A Tabela 1 apresenta a identificação dos materiais estudados, a composição C1 é de UHMWPE, C2 é UHMWPE com adição de 10\% em massa de LLDPE e C3 é UHMWPE com adição de $20 \%$ em massa de LLDPE .

Tabela 1: Identificação dos compósitos.

\begin{tabular}{c|c}
\hline IDENTIFICAÇÃo & COMPOSIÇÃO \\
\hline C1 & UHMWPE \\
\hline C2 & UHMWPE/LLDPE (90/10) \\
\hline C3 & UHMWPE / LLDPE (80/20) \\
\hline
\end{tabular}

Além disso, cada identificação é seguida pela quantidade de nanotubos de carbono adicionado ( $0 ; 0,5$; 1,0 e 2,0 \% em massa), assim, por exemplo, a composição C1-0,5 corresponde ao PEAUMM com 0,5\% em massa de nanotubos de carbono.

\subsection{Caracterização mecânica das blendas e nanocompósitos poliméricos}

A caracterização mecânica das amostras de blendas e nanocompósitos foi feita através de ensaio de tração uniaxial utilizando uma máquina de Ensaio Universal Instron, modelo $430 \mathrm{com}$ velocidade de subida da travessa de $50 \mathrm{~mm} \cdot \mathrm{min}^{-1}$ e utilizando uma célula de carga de $5 \mathrm{kN}$. Foram testados 5 corpos de prova de cada composição.

\subsection{Caracterização térmica das blendas e nanocompósitos poliméricos}

As blendas e os nanocompósitos poliméricos foram caracterizados através de calorimetria exploratória diferencial (DSC) utilizando um equipamento da TA Instruments, modelo QS100 com fluxo contínuo de 50 ml.min-1 de gás nitrogênio. As amostras foram inicialmente aquecidas da temperatura ambiente até $180{ }^{\circ} \mathrm{C}$ a uma taxa de aquecimento de $10{ }^{\circ} \mathrm{C} \cdot \mathrm{min}^{-1}$, seguida de isoterma por 3 minutos. A seguir foram resfriadas até $40{ }^{\circ} \mathrm{C}$ a uma taxa de $10{ }^{\circ} \mathrm{C} \cdot \min ^{-1}$ para determinação da temperatura de cristalização (Tc) e novamente foram aquecidas até $180^{\circ} \mathrm{C}$ a uma taxa de $10^{\circ} \mathrm{C} \cdot \mathrm{min}^{-1}$. A temperatura de fusão cristalina (Tm) e a entalpia de fusão foram obtidas no segundo ciclo de aquecimento. A estabilidade térmica dos materiais desenvolvidos foi avaliada através de análise termogravimétrica (TGA) utilizando um equipamento da Netzsch, modelo 209 F1, a uma taxa de aquecimento de $20{ }^{\circ} \mathrm{C} \cdot \mathrm{min}^{-1}$, da temperatura ambiente até $800^{\circ} \mathrm{C}$, sob fluxo constante de 20 $\mathrm{ml} \cdot \mathrm{min}^{-1}$ de gás nitrogênio.

\subsection{Caracterização morfológica}

A caracterização morfológica dos nanocompósitos foi realizada utilizando um microscópio eletrônico de varredura FEG-MEV Mira 3 Tescan operando com $10 \mathrm{kV}$. As amostras foram congeladas em nitrogênio líquido e fraturadas, em seguida as superfícies receberam uma fina deposição de ouro.

\subsection{Caracterização dos CNT}

Os CNT com funcionalização e sem funcionalização foram avaliados por espectroscopia Raman, em um espectrofotômetro da marca Renishaw Microscopy System modelo 2000 usando uma fonte de excitação de laser com potência $2,41 \mathrm{eV}$ e comprimento de onda de $514,5 \mathrm{~nm}$ na região de 300 a $2400 \mathrm{~cm}^{-1}$ e por ensaio termogravimétrico em um equipamento da Netzsch, modelo $209 \mathrm{~F} 1$ da temperatura ambiente até $1000{ }^{\circ} \mathrm{C}$ sob atmosfera inerte de nitrogênio com fluxo de $100 \mathrm{ml} \cdot \mathrm{min}^{-1}$ e em atmosfera oxidante de ar sintético com fluxo de $20 \mathrm{ml} \cdot \mathrm{min}^{-1}$. 


\section{RESULTADOS}

\subsection{Caracterização mecânica das blendas e nanocompósitos}

A Figura 1 apresenta as curvas obtidas por tração uniaxial das blendas poliméricas e dos nanocompósitos estudados.
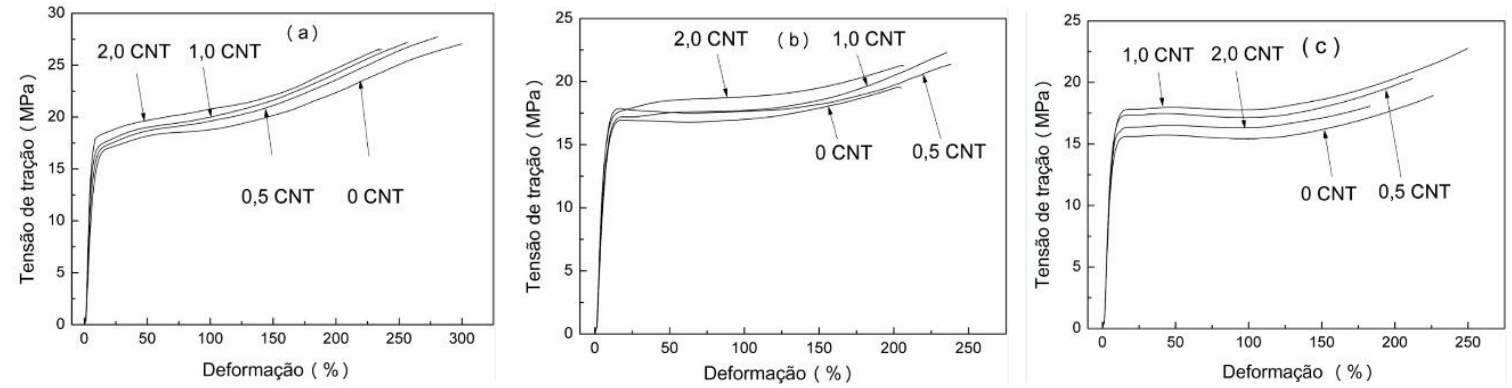

Figura 1: Curvas tensão versus deformação obtidas pelo ensaio de tração uniaxial dos nanocompósitos com os diferentes teores de nanotubos de carbono (a) $\mathrm{C} 1$, (b) $\mathrm{C} 2$ e (c) $\mathrm{C} 3$.

A Tabela 2 apresenta os valores de dureza e das propriedades mecânicas obtidas pelo ensaio de tração uniaxial das blendas e nanocompósitos estudados com diferentes teores de LLDPE e CNT estudados, são apresentados a média dos valores obtidos e os respectivos desvios padrão.

Tabela 2: Propriedades mecânicas obtidas pelo ensaio de tração uniaxial e dureza das composições estudadas.

\begin{tabular}{l|l|l|l|l|l|l}
\hline AMOSTRA & $\boldsymbol{\sigma}_{\mathrm{e}}(\mathbf{M P a})$ & $\boldsymbol{\sigma}_{\mathbf{v}}(\mathbf{M P a})$ & $\mathbf{E}(\mathbf{M P a})$ & $\mathbf{A L}(\%)$ & $\mathbf{J}\left(\mathbf{M J} / \mathbf{m}^{3}\right)$ & Shore(D) \\
\hline C1-0 & $8,5 \pm 0,4$ & $74 \pm 12$ & $352 \pm 9$ & $292 \pm 38$ & $48 \pm 5$ & $68 \pm 2$ \\
\hline C1-0,5 & $8,9 \pm 0,4$ & $70 \pm 12$ & $355 \pm 8$ & $273 \pm 31$ & $54 \pm 7$ & $73 \pm 2$ \\
\hline C1-1 & $9,9 \pm 0,2$ & $63 \pm 8$ & $372 \pm 23$ & $254 \pm 28$ & $52 \pm 4$ & $77 \pm 1$ \\
\hline C1-2 & $9,8 \pm 0,2$ & $70 \pm 4$ & $406 \pm 12$ & $246 \pm 27$ & $46 \pm 5$ & $75 \pm 2$ \\
\hline C2-0 & $8,2 \pm 0,4$ & $58 \pm 16$ & $364 \pm 7$ & $225 \pm 33$ & $38 \pm 4$ & $69 \pm 5$ \\
\hline C2-0,5 & $9,2 \pm 0,4$ & $57 \pm 9$ & $350 \pm 15$ & $238 \pm 25$ & $47 \pm 7$ & $71 \pm 2$ \\
\hline C2-1 & $9,5 \pm 0,2$ & $58 \pm 7$ & $360 \pm 11$ & $235 \pm 21$ & $48 \pm 7$ & $72 \pm 2$ \\
\hline C2-2 & $9,4 \pm 0,3$ & $54 \pm 4$ & $384 \pm 8$ & $227 \pm 26$ & $42 \pm 6$ & $72 \pm 2$ \\
\hline C3-0 & $8,0 \pm 0,1$ & $54 \pm 4$ & $344 \pm 16$ & $226 \pm 24$ & $32 \pm 4$ & $68 \pm 2$ \\
\hline C3-0,5 & $9,0 \pm 0,1$ & $52 \pm 4$ & $350 \pm 8$ & $212 \pm 21$ & $38 \pm 5$ & $71 \pm 5$ \\
\hline C3-1 & $9,2 \pm 0,2$ & $60 \pm 3$ & $370 \pm 6$ & $248 \pm 10$ & $44 \pm 5$ & $71 \pm 1$ \\
\hline C3-2 & $9,2 \pm 0,1$ & $43 \pm 3$ & $384 \pm 17$ & $183 \pm 13$ & $30 \pm 4$ & $68 \pm 2$ \\
\hline
\end{tabular}

Onde: $\sigma e:$ é a tensão de escoamento, $\sigma \mathrm{v}$ : tensão máxima verdadeira, E: módulo elástico, AL: alongamento na ruptura e J: energia absorvida até a ruptura. A tensão máxima verdadeira foi obtida através de cálculo utilizando a seção transversal dos corpos de prova após ruptura.

\subsection{Caracterização térmica das blendas e nanocompósitos poliméricos}

A Figura 2 apresenta as curvas termogravimétricas dos nanocompósitos C1, C2 e C3 com os diferentes teores de nanotubos de carbono, as temperaturas de início de degradação térmica destes materiais são mostradas na Tabela 3. 

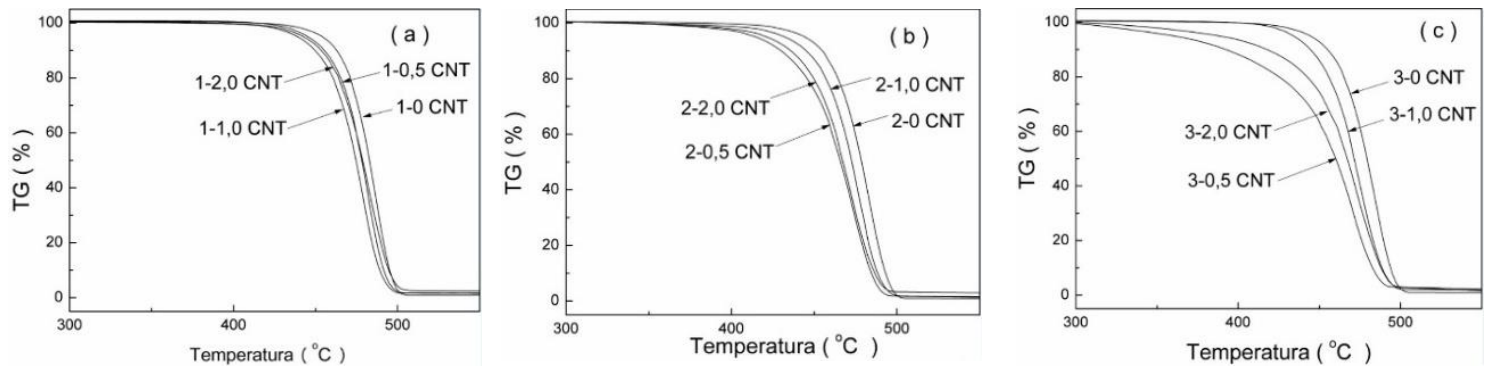

Figura 2: Curvas obtidas pela análise termogravimétrica dos nanocompósitos com os diferentes teores de nanotubos de carbono (a) C1, (b) C2 e (c) C3.

A Figura 3 apresenta os termogramas de DSC dos nanocompósitos C1 com os diferentes teores de CNT durante o resfriamento e o segundo ciclo de aquecimento, a Tabela 3 apresenta os parâmetros térmicos.
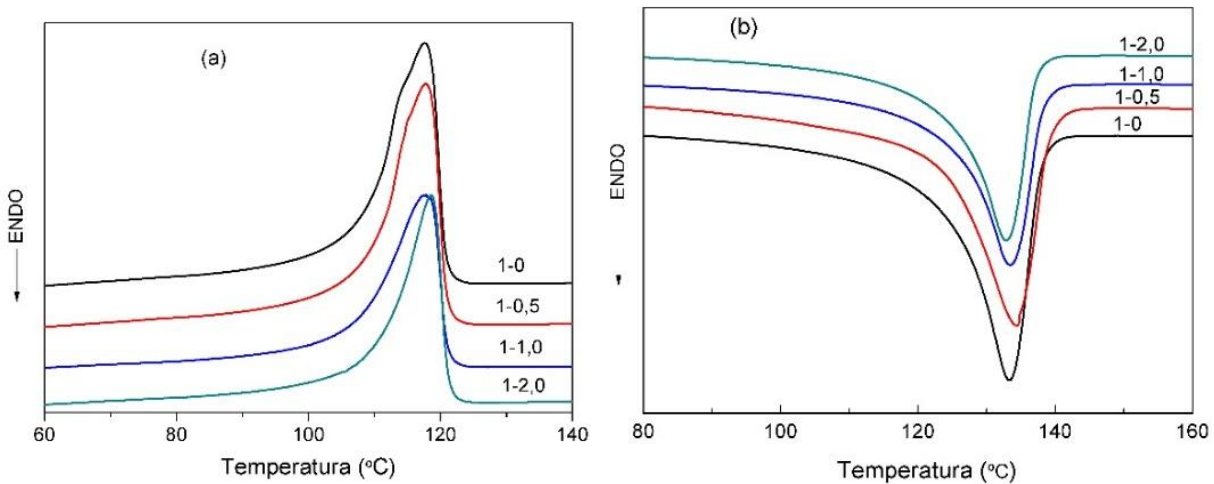

Figura 3: Termogramas de DSC dos nanocompósitos $\mathrm{C} 1$ com os diferentes teores de nanotubos de carbono (a) durante o resfriamento (b) durante o aquecimento.

A Figura 4 apresenta os termogramas de DSC dos nanocompósitos C2 com os diferentes teores de CNT durante o resfriamento e o segundo ciclo de aquecimento, a Tabela 3 apresenta os parâmetros térmicos.
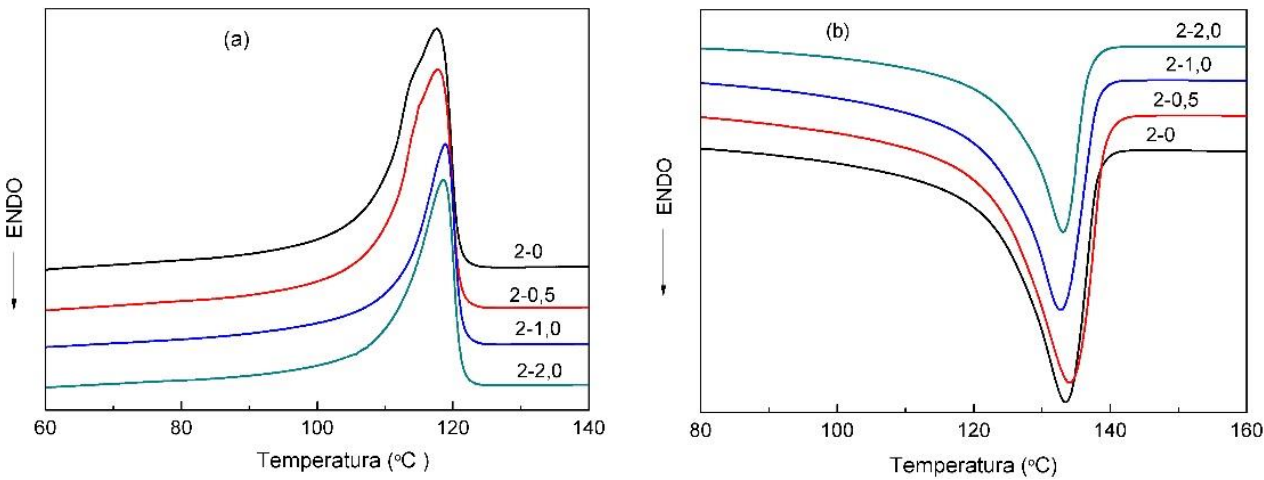

Figura 4: Termogramas de DSC dos nanocompósitos C2 com os diferentes teores de nanotubos de carbono (a) durante o resfriamento (b) durante o aquecimento.

A Figura 5 apresenta os termogramas de DSC dos nanocompósitos C3 com os diferentes teores de CNT durante o resfriamento e o segundo ciclo de aquecimento, a Tabela 3 apresenta os parâmetros térmicos. 

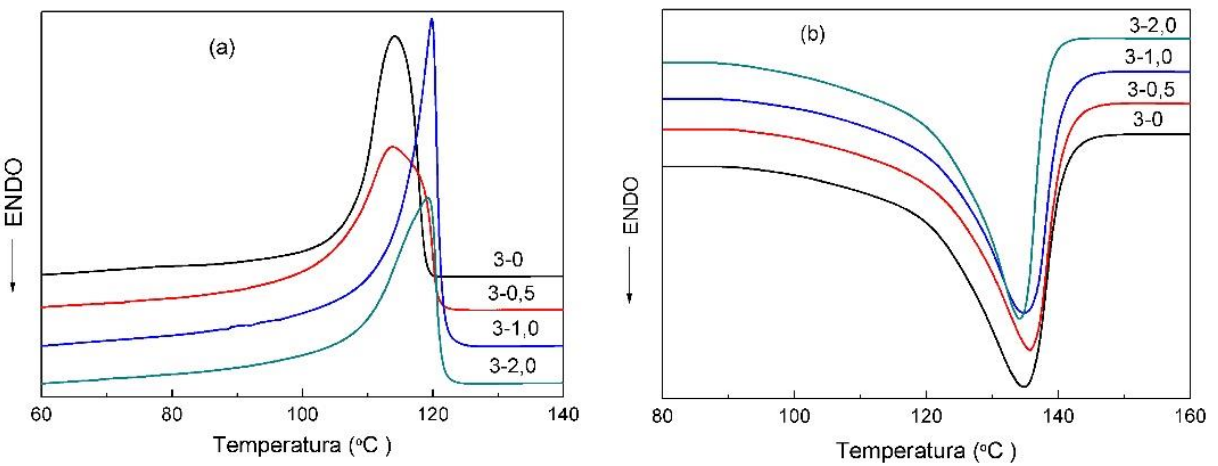

Figura 5: Termogramas de DSC dos nanocompósitos C3 com os diferentes teores de nanotubos de carbono (a) durante o resfriamento (b) durante o aquecimento.

A Tabela 3 apresenta os valores das propriedades térmicas das blendas e nanocompósitos estudados.

Tabela 3: Propriedades térmicas das blendas e nanocompósitos poliméricos obtidos por TGA e DSC.

\begin{tabular}{l|l|l|l|l|l}
\hline AMOSTRA & TG-Onset & $\mathbf{T c}\left({ }^{\circ} \mathbf{C}\right)$ & $\mathrm{Tm}_{2}\left({ }^{\circ} \mathbf{C}\right)$ & $\Delta \mathbf{H m}_{2}(\mathbf{J} / \mathbf{g})$ & $\mathbf{X}_{2}(\%)$ \\
\hline C1-0 & 470 & 118 & 134 & 134,1 & 49,4 \\
\hline C1-0,5 & 469 & 118 & 134 & 129,0 & 47,3 \\
\hline C1-1 & 461 & 117 & 134 & 124,9 & 46,0 \\
\hline C1-2 & 455 & 118 & 135 & 124,0 & 45,6 \\
\hline C2-0 & 464 & 116 & 133 & 143,9 & 53,0 \\
\hline C2-0,5 & 456 & 115 & 134 & 140,4 & 51,7 \\
\hline C2-1 & 459 & 117 & 133 & 137,9 & 50,8 \\
\hline C2-2 & 460 & 118 & 133 & 129,9 & 47,8 \\
\hline C3-0 & 464 & 116 & 134 & 129,4 & 50,3 \\
\hline C3-0,5 & 456 & 113 & 136 & 123,0 & 47,8 \\
\hline C3-1 & 459 & 119 & 135 & 125,7 & 48,9 \\
\hline C3-2 & 454 & 117 & 134 & 133,5 & 51,9 \\
\hline
\end{tabular}

Onde: TG-Onset: é a temperatura de início da degradação térmica, Tc: temperatura de cristalização medida no ciclo de resfriamento, $\mathrm{Tm}_{2}$ : temperatura de fusão cristalina medida no segundo ciclo de aquecimento, $\Delta \mathrm{Hm}_{2}$ : energia de entalpia de fusão cristalina medida no segundo ciclo de aquecimento e $\mathrm{Xc}_{2}$ : grau de cristalinidade calculado.

O grau de cristalinidade dos polímeros foi determinado utilizando a equação 1:

$$
X c(\%)=\frac{\Delta H m-\Delta H c}{\Delta H m^{\circ}} \times 100 \%
$$

Onde: Xc é a porcentagem de cristalinidade, $\Delta \mathrm{Hm}$ é a entalpia de fusão cristalina da amostra obtida por DSC, $\triangle \mathrm{Hc}$ é a entalpia de cristalização ocorrida durante o aquecimento, neste caso o valor é zero, e o $\Delta \mathrm{Hm}^{\mathrm{o}}$ é a entalpia de fusão de equilíbrio para o material teoricamente $100 \%$ cristalino.

$\mathrm{O}$ valor de $\Delta \mathrm{Hm}^{\mathrm{o}}$ das blendas foi calculado através da equação 2, obtendo 271,6 J/g para o nanocompósito 2 (90/10) e 257,1 J/g para o nanocompósito $3(80 / 20)$.

$$
\Delta H m^{o} \text { blenda }=\frac{\Delta H m_{a}^{o} \cdot x_{a}+\Delta H m_{b}^{o} \cdot x_{b}}{x_{a}+x_{b}}
$$

Onde: o valor de $\Delta \mathrm{Hm}_{\mathrm{a}}^{\mathrm{o}}$ (UHMWPE) é de $286,2 \mathrm{~J} / \mathrm{g}$ e para o $\Delta \mathrm{Hm}_{\mathrm{b}}^{\mathrm{o}}$ (LLDPE) é de 140,6 J/g [20], $\mathrm{x}_{\mathrm{a}}$ é a fração em massa do UHMWPE e $\mathrm{x}_{\mathrm{b}}$ a fração em massa do LLDPE. 


\subsection{Caracterização morfológica dos nanocompósitos poliméricos}

A Figura 6 apresenta imagens obtidas por FEG-MEV de diferentes superfícies criofraturadas do nanocompósito $\mathrm{C} 1 \mathrm{com} 2 \%$ em massa de nanotubos de carbono $(\mathrm{C} 1-2,0)$.
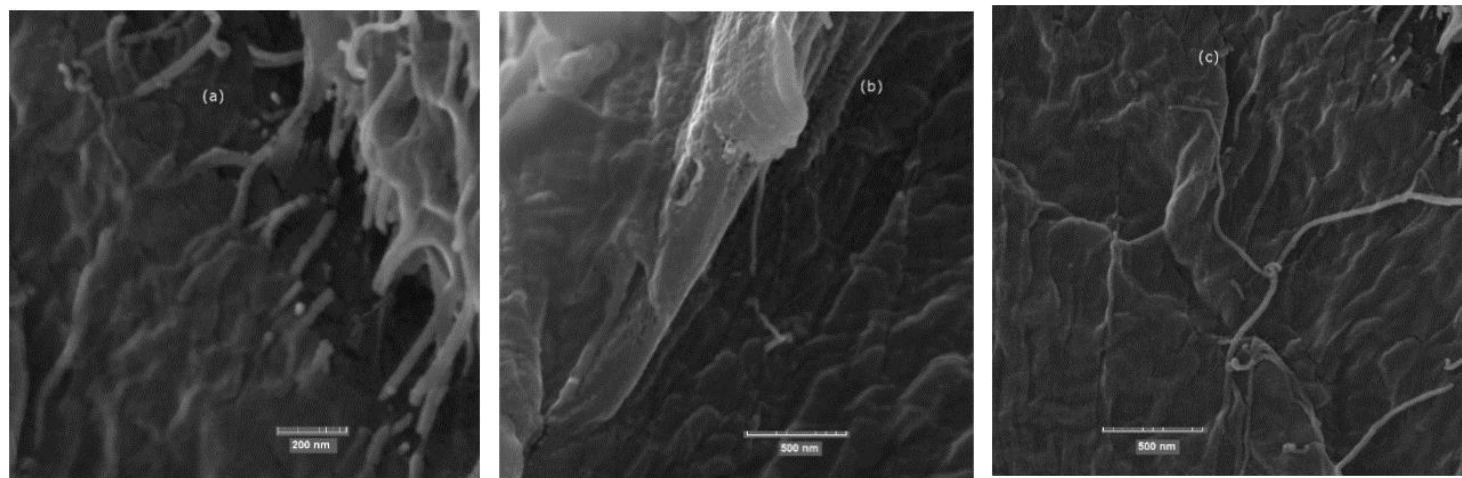

Figura 6: Micrografias de FEG-MEV do nanocompósito C1-2,0 (a) com ampliação de 200.000 x, (b) e (c) com ampliação de 100.000 x

A Figura 7 apresenta imagens de FEG-MEV de diferentes superfícies criofraturadas do nanocompósito $\mathrm{C} 2$ com $1 \%$ em massa de nanotubos de carbono $(\mathrm{C} 2-1,0)$.
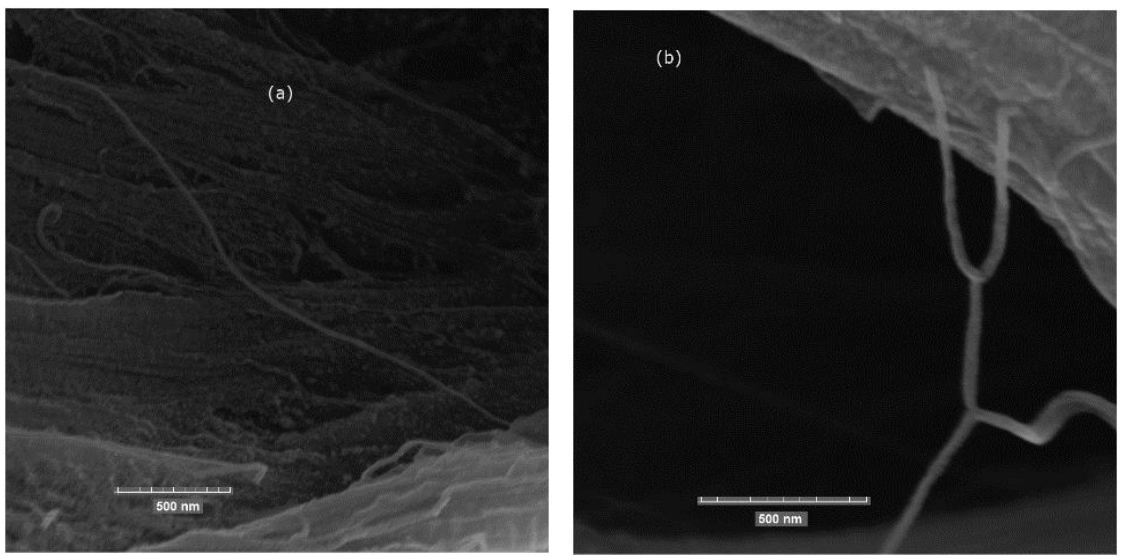

Figura 7: Micrografias de FEG-MEV do nanocompósito C2-1,0 (a) com ampliação de 100.000 x e (b) com ampliação de $150.000 \mathrm{x}$.

A Figura 8 apresenta micrografia de FEG-MEV do nanocompósito C3 com $1 \%$ de nanotubos de carbono $(\mathrm{C} 3-1,0)$.
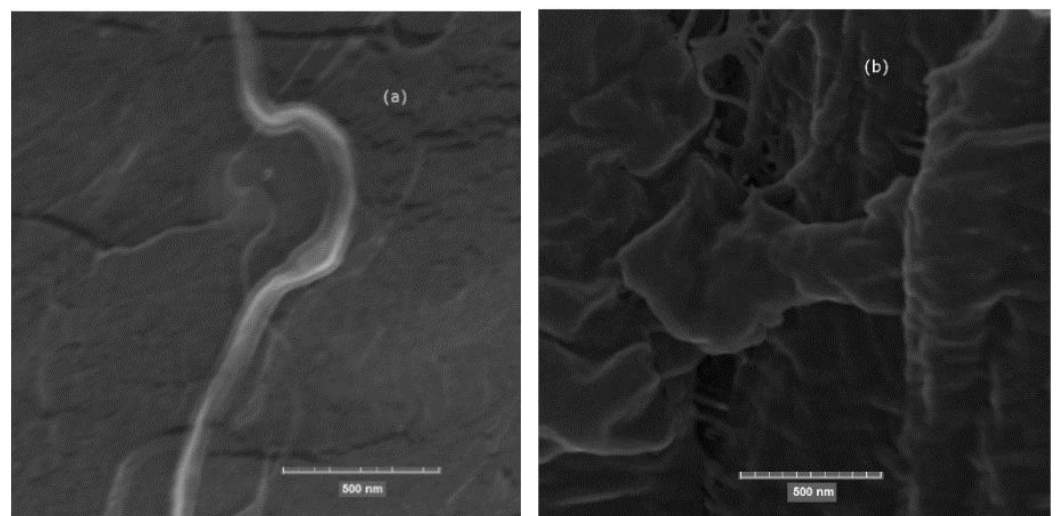

Figura 8: Micrografias de FEG-MEV do nanocompósito C3-1,0 com ampliação de 200.000 x. 
A Figura 9 apresenta as curvas de espectroscopia Raman (a) e em (b) as curvas de análise termogravimétrica dos CNT submetidos ao tratamento superficial e sem tratamento.
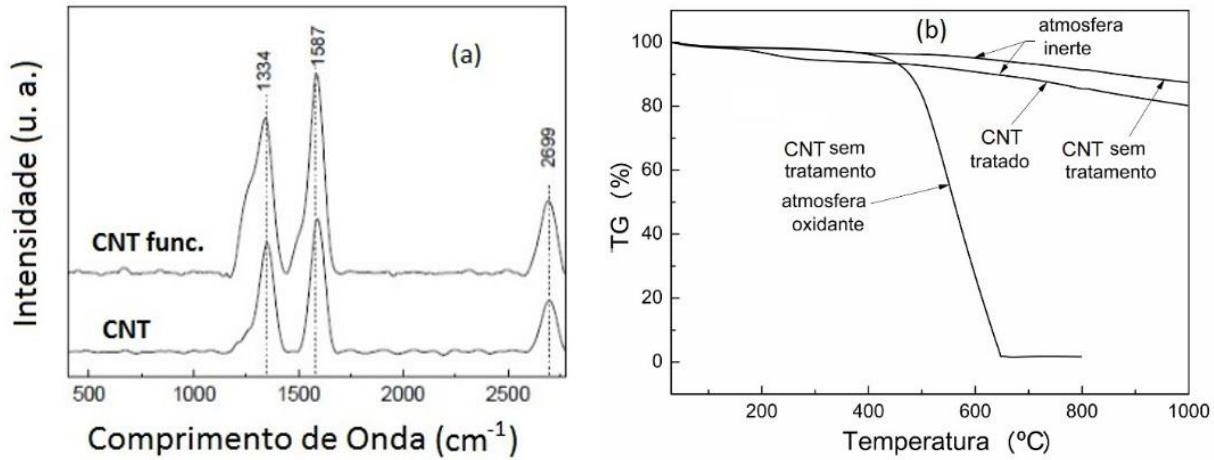

Figura 9: Avaliação dos CNT tratados e sem tratamento, por espectroscopia Raman (a) e análise termogravimétrica (b).

\section{DISCUSSÃO}

Na Figura 6 é possível observar a presença de nanotubos de carbono rompidos (efeito ponte) nas imagens (a) e (b) e a formação de entrelaçamentos entre nanotubos na imagem (c). Na Figura 7 é possível observar na imagem (a) a presença de um CNT longo solto da matriz polimérica e vários CNT presentes na matriz e na imagem (b) é possível observar a formação de união entre nanotubos de carbono com as extremidades fixadas na matriz polimérica. Na Figura 8 (a) é possível observar a presença de um nanotubo de carbono de múltiplas paredes na superfície da matriz polimérica.

A Figura 9 (a) apresenta as bandas características D e G em $1334 \mathrm{~cm}^{-1}$ e $1587 \mathrm{~cm}^{-1}$ e uma banda de segunda ordem G' em $2694 \mathrm{~cm}^{-1}$, para os CNT funcionalizados e bandas em $1352 \mathrm{~cm}^{-1}, 1591 \mathrm{~cm}^{-1}$ e $2699 \mathrm{~cm}^{-}$ ${ }^{1}$ respectivamente para os CNT sem tratamento. O modo de banda G no CNT sem tratamento foi encontrado em $1591 \mathrm{~cm}^{-1}$, enquanto a literatura indica em $1590 \mathrm{~cm}^{-1}$. Este modo é o de vibração tangencial do carbono grafítico (grafite). Isso indica que os CNT utilizados apresentam relativo baixo teor de impurezas, o que é consistente com a especificação de pureza do fabricante de $95 \%$ e pelo ensaio de TGA que apresentou apenas $2 \%$ de massa residual em atmosfera oxidante, Figura 9 (b). O desvio das bandas registradas é uma indicação de defeitos de rede causados pelo tratamento com ácido nítrico [21]. Na Figura 9 (b) pode ser observado que o CNT tratado com ácido nítrico em atmosfera inerte tem uma perda de massa inicial entre 200 e $300^{\circ} \mathrm{C}$ que corresponde ao grupo carboxílico e uma segunda perda de massa a partir de $450^{\circ} \mathrm{C}$ que provavelmente pertença ao grupo de anidrido carboxílico [22]. O CNT sem tratamento e em atmosfera inerte é mais estável termicamente, começa a degradação a partir de $550{ }^{\circ} \mathrm{C}$. O CNT sem tratamento e sob atmosfera oxidante apresenta grande perda de massa a partir de $400^{\circ} \mathrm{C}$ e degradação máxima em $650^{\circ} \mathrm{C}$.

A adição de nanotubos de carbono funcionalizados no UHMWPE aumentou o módulo elástico e a tensão de escoamento do nanocompósito. Quanto maior a quantidade de nanotubos de carbono adicionado, observou-se maiores valores de módulo elástico. Como consequência do aumento da rigidez das composições observou-se uma ligeira redução na deformação até a ruptura. O maior aumento do módulo de Young foi observado nos nanocompósitos com 2,0 \% em massa de nanotubos de carbono.

A adição de $10 \%$ em massa de LLDPE no UHMWPE ocasionou um pequeno aumento no módulo elástico da blenda polimérica em relação ao UHMWPE puro, no entanto, aumentando-se esse teor para $20 \%$ em massa de LLDPE ocorreu a diminuição do módulo elástico, notadamente pela menor resistência mecânica do LLDPE. Em todos os casos, notou-se que a adição dos nanotubos de carbono ocasionaram aumento significativo no módulo elástico. Nas micrografias é possível observar a presença dos nanotubos de carbono atuando como reforço na matriz polimérica. Vale ressaltar que a adição da segunda fase (LLDPE) é fundamental para obtenção do material fundido/amolecido pela técnica de processamento utilizada. Por essa técnica, ocorre fusão/amolecimento do material decorrente do aquecimento viscoso ocasionado pelo atrito. O homogeneizador do tipo DRAIS com rotor possui velocidade de $3000 \mathrm{rpm}$, ocorrendo aumento da temperatura da massa processada decorrente do atrito gerado entre partículas e entre partícula-rotor. O LLDPE auxilia no processo de mistura, uma vez que possui viscosidade do fundido relativamente baixa (possui índice de fluidez de 29 
g.10 $\left.\min ^{-1}\left(190{ }^{\circ} \mathrm{C} / 2,16 \mathrm{Kg}\right)\right)$ e proporciona melhor mistura com o UHMWPE, contribuindo para diminuição da viscosidade do fundido da mistura polimérica e facilitando esse processo.

Neste estudo é proposto um novo método de mistura para obtenção das placas poliméricas para uso balístico, visando a utilização de equipamentos que proporcione uma maior quantidade de material produzido. A adição de $20 \%$ em massa de LLDPE na composição C3 possui a maior facilidade de mistura, sendo melhor misturados e homogeneizados e o tempo de mistura reduzido, no entanto, o aumento de LLDPE na composição ocasionou redução do módulo elástico comparado ao UHMWPE puro. A alternativa de adição de nanotubos de carbono na composição para compensar a perda de módulo elástico decorrente da adição da segunda fase (LLDPE) mostrou-se bastante satisfatória obtendo-se valores superiores ao UHMWPE puro quando adicionado 1,0 \% em massa de CNT na composição das blendas UHMWPE/LLDPE (90/10 e 80/20).

O maior módulo elástico das composições pode estar relacionado com a maior rigidez das composições, para todas as composições desenvolvidas observou-se um aumento na dureza com a adição dos nanotubos de carbono e, aumentando-se a quantidade de CNT nas composições, ocorre um subsequente aumento na dureza (Tabela 2). Zoo et al. [23] também observou aumento da dureza (microdureza Vickers) nos nanocompósitos de UHMWPE com o aumento do teor de CNT $(0,1 ; 0,2$ e $0,5 \%$ em massa), corroborando com os resultados obtidos nesse trabalho.

As principais propriedades do material polimérico para uso balístico é a capacidade de absorção de energia e resistência mecânica. Na absorção de energia o nanocompósito C2-1,0 atingiu o mesmo valor do UHMWPE puro (48 MJ/m³ $)$ e na tensão de escoamento foi 11,7\% superior ao UHMWPE puro (Tabela 2). A absorção de energia ou tenacidade pode ser estimada através da medida da área abaixo da curva tensão versus deformação até a fratura do material. Utilizando-se dessa teoria, observou-se que a adição de 0,5 e 1,0 \% em massa de CNT melhorou esta propriedade para todos os nanocompósitos estudados.

Khan et al. [24] em seus estudos de absorção de energia de filamentos obteve o valor de $41 \mathrm{MJ} / \mathrm{m}^{3}$ para UHMWPE puro e de $48 \mathrm{MJ} / \mathrm{m}^{3}$ para UHMWPE com $2 \%$ em massa de nanotubos de carbono com parede única, valores similares aos encontrados nesse trabalho, mostrando que o processo de mistura adotado não prejudica as propriedades dos nanocompósitos desenvolvidos.

Assim como neste trabalho, outros pesquisadores dispersaram com sucesso nanotubos de carbono em polímeros termoplásticos e demonstraram que a tensão e o módulo de elasticidade poderiam ser melhorados, entretanto, essas melhorias são obtidas com uma diminuição significativa da deformação na ruptura, que eventualmente diminuiu a capacidade de absorção de energia. Khan et al. [24,25] observaram que a adição de LDPE como segunda fase polimérica pode melhorar a deformação na ruptura e a tenacidade à fratura do nanocompósito de UHMWPE/CNT.

No que concerne às propriedades térmicas, notou-se que a adição da segunda fase polimérica e dos nanotubos de carbono apresentaram pouca influência nas temperaturas de fusão cristalina e temperaturas de cristalização das blendas poliméricas e nanocompósitos. Nota-se, no entanto, que apenas uma temperatura de fusão cristalina e uma temperatura de cristalização foi observada para as blendas poliméricas com 10 e $20 \%$ em massa de LLDPE e para seus respectivos nanocompósitos. Esse fato é um indicativo da miscibilidade dessa blenda polimérica e sugere que esteja ocorrendo co-cristalização do UHMWPE com o LLDPE. Na cocristalização, os dois polímeros formam cristais isomórficos, ou seja, as cadeias de ambos os polímeros participam na formação do cristalito. A possibilidade de formação de blendas de polietileno que apresentam cocristalização depende principalmente do número e do tamanho das ramificações. Segundo Choi [26] a separação de fases pode ocorrer quando a quantidade de ramificações for igual ou superior a 40 ramificações por 1000 átomos de carbono. Nesse caso, ambos polímeros são lineares e com baixo teor de ramificação, fortalecendo a hipótese de ocorrência de co-cristalização.

Analisando os valores de grau de cristalinidade das composições, observa-se que a adição de nanotubos de carbono diminui o grau de cristalinidade dos nanocompósitos. A dificuldade da ordenação das cadeias poliméricas com adição dos nanotubos de carbono pode estar relacionado com o elevado comprimento dos CNT utilizados.

Zoo et al. [23] observaram uma leve diminuição no grau de cristalinidade e aumento do módulo elástico dos nanocompósitos de UHMWPE com o aumento do teor de nanotubos de carbono, até 0,5\% em massa (diâmetro de 10-50 nm e comprimento de 3-5 $\mu \mathrm{m}$ ), justificaram que os CNT atuaram como reforço da matriz polimérica, mas sem afetar a estrutura interna do UHMWPE. Por outro lado, Khan et al. [24] observaram um aumento no grau de cristalinidade do nanocompósito de UHMWPE com adição de $2 \%$ em massa de nanotubos de carbono de parede única (diâmetro de $2-10 \mathrm{~nm}$ e comprimento de $1-2 \mu \mathrm{m}$ ).

Comparando os valores de grau de cristalinidade das blendas poliméricas com 10 e $20 \%$ em massa de LLDPE nota-se o aumento no grau de cristalinidade em comparação ao UHMWPE puro, uma vez que o 
LLDPE possui menor viscosidade do fundido e participa da formação do cristalito (co-cristalização), sua presença facilita a ordenação das cadeias poliméricas ocasionando aumento no grau de cristalinidade.

A temperatura de início da degradação térmica irreversível (TG-onset) foi levemente afetada pela adição da segunda fase e das nanocargas, houve uma diminuição na temperatura de degradação com o aumento do teor de LLDPE e de CNT. O LLDPE possui menor estabilidade térmica, devido ao menor tamanho de cadeia polimérica e a presença desse componente ocasiona diminuição nesse parâmetro. A adição de CNT diminui o grau de cristalinidade e ordenamento molecular das cadeias de UHMWPE, logo menos energia é necessária para que ocorra a degradação térmica, ocasionando diminuição desse parâmetro com a adição e aumento do teor de CNT nas composições.

\section{CONCLUSÕES}

A adição de nanotubos de carbono funcionalizados com ácido nítrico até o teor de 1,0 \% em massa na blenda polimérica de UHMWPE/LLDPE obtidos por mistura mecânica em homogeneizador do tipo DRAIS obteve uma melhora significativa nas propriedades mecânicas dos compósitos poliméricos. Os nanocompósitos com 2,0 \% em massa de CNT obtiveram os maiores valores de módulo elástico, entretanto houve queda da deformação e da absorção de energia. Os CNT atuaram fortemente como reforço da matriz polimérica, com boa interface entre nanotubos de carbono e matriz polimérica. A adição da segunda fase (LLDPE) na matriz de UHMWPE foi fundamental para obtenção do material fundido/amolecido pela técnica de processamento utilizada e junto com a adição de CNT os nanocompósitos apresentaram propriedades mecânicas e térmicas semelhantes ao UHMWPE puro, tendo a vantagem da facilidade de processamento.

Com relação à estabilidade térmica houve uma sutil redução, fato que não afeta o desempenho balístico, visto que a faixa de aplicação da blindagem é de até $60{ }^{\circ} \mathrm{C}$, bem inferior à temperatura de início da degradação térmica apresentada pelos compósitos (454-470 $\left.{ }^{\circ} \mathrm{C}\right)$. O grau de cristalinidade dos compósitos diminuiu com o aumento do teor de CNT e aumentou com a adição da segunda fase em comparação ao UHMWPE puro. As blendas desenvolvidas são miscíveis.

O nanocompósito de UHMWPE/LLDPE (80/20) com adição de 1,0 \% em massa de CNT apresentouse como uma solução viável para produção de placas poliméricas em larga escala utilizando o processo proposto para fabricação de proteção balística.

\section{AGRADECIMENTOS}

Os autores gostariam de agradecer à FAPESP (2014/04900-9) e CNPq (442081/2014-6) pelo suporte financeiro.

\section{BIBLIOGRAFIA}

[1] SILVA, M.V., STAINER, D., Al-QURESHI, H.A., et al., "Alumina-based ceramics for armor application: mechanical characterization and ballistic testing”, Journal of Ceramics, v. 2014, pp.1-6, ID 618154, 2014.

[2] CAREY, M. E., "Analysis of wounds incurred by US Army Seventh Corps personnel treated in Corps hospitals during Operation Desert Storm”, The Journal of trauma, v. 40, 3 Suppl, pp. 165-169, 1996.

[3] KAUFMANN, C., CRONIN, D., WORSWICK, M., et al., "Influence of material properties on the ballistic performance of ceramics for personal body armour", Shock and Vibration, v. 10, pp. 51-58, 2003.

[4] DEPLANCKE, T., LAME, O., ROUSSET, F., et al., "Diffusion versus cocrystallization of very long polymer chains at interfaces: experimental study of sintering of UHMWPE nascent powder",

Macromolecules, v. 47, n. 1, pp. 197-207, 2013.

[5] HAMBIR, S., JOG, J. P., "Sintering of ultra high molecular weight polyethylene", Bulletin of Materials Science, v. 23, pp. 221-226, 2000.

[6] LIU, Y., SINHA, S. K., LIM, C. Y. H, et al., "Pre-polishing the metal counterface of metal-UHMWPE wear pair with filler-filled UHMWPE composites to generate counterface changes for an effective reduction in pure UHMWPE wear", Tribology Letters, v. 53, pp.11-16, 2014.

[7] PREVORSEK, D.C., KWON, Y. D., CHIN, H. B., “Analysis of the temperature rise in the projectile and extended chain polyethylene fiber composite armor during ballistic impact and penetration", Polymer

Engineering Science, v. 34, pp. 141-152, 1994. 
[8] LEE, B. L., WALSH, T. F., WON, S.T., et al, "Penetration failure mechanisms of armor-grade fiber composites under impact”, Journal of Composites Materials, v. 35, pp. 1605-1635, 2001.

[9] ICHIKAWA, K.U., DEPLANCKE, T., OGAWA, K., et al, "Development of ultra-high molecular weight polyethylene (UHMWPE) coating by cold spray technique", Journal of Thermal Spray Technology, v. 24, n. 6, pp. 1015-1025, jul. 2015.

[10] ABRAHAM, D., GEORGE, K. E., FRANCIS, D. J., "Rheological caracterization of blends of low density with linear low density polyethylene using a torque rheometer", European Polymer Journal, v. 26, n. 2, pp. 197-200, 1990.

[11] COUTINHO, F. M. B., MELLO, I. L., MARIA, L. C., "Polietileno: principais tipos, propriedades e aplicações", Polímeros: Ciência e Tecnologia, v. 13, pp.1-13, 2003.

[12] PEACOCK, A. J., Handbook of Polyethylene: Structures, Properties and Applications, New York: Marcel Dekker, 534 pp., 2000.

[13] HAGE, E., PESSAN, L. A., Aperfeiçoamento em Tecnologia de plásticos, Apostila Módulo 7: Blendas Poliméricas, 142 p., Universidade Federal de São Carlos, São Carlos, 2001.

[14] ZHANG, X.D., TANAKA, J., YU, Y.T., et al, "Wear of UHMWPE against zirconia/alumina composite”, Key Eng Mater, v. 625, pp. 288-289, 2005.

[15] XU, S., TANGPONG, X. W., "Review: Tribological behavior of polyethylene-based nanocomposites”, Journal of Material Science, v. 48, pp. 578-597, 2013.

[16] CHENG, Q., WANG, J., JIANG, K., et al, "Fabrication and properties of aligned mutiwalled carbon nanotube-reinforced epoxy composites”, Journal of Materials Resistance, v. 23, pp. 2975-2983, 2008.

[17] GARCIA, E. J., HART, A. J., WARDLE, B.L., et al., "Fabrication of composite microstructures by capillarity-driven wetting of aligned carbon nanotubes with polymers", Nanotechnology, v. 18, 2007.

[18] MAJUMDER, M., CHOPRA, N., ANDREWS, R., et al., "Nanoscale hydrodynamics: Enhanced flow in carbon nanotubes", Nature, v. 438, dec. 2005.

[19] ZHANG, S.C., FAHRENHOLTZ, W.G., HILMAS, G.E., et al., "Pressureless sintering of carbon nanotube- $\mathrm{Al}_{2} \mathrm{O}_{3}$ composites", Journal of the European Ceramic Society, v. 30, pp. 1373-1380, 2010.

[20] WUNDERLICH, B., Macromolecular Physics, New York: Academic Press, v. 3, Crystal Melting, 1980.

[21] DATSYUK, V., KALYVA, M., PAPAGELIS, K., et al. "Chemical oxidation of multiwalled carbon nanotube", Carbon, v.46, pp. 833-840, 2008.

[22] FIGUEIREDO, J.L., PEREIRA, M.F.R., FREITAS, M.M.A., et al., "Modification of the surface chemistry of activated carbons", Carbon, v. 37, pp. 1379-1389, 1999.

[23] ZOO, Y.S., AN, J.W., LIM, D.P., et al., "Effect of carbon nanotube addition on tribological behavior of UHMWPE", Tribology Letters, vol.16, no. 4, 2004.

[24] M.R.KHAN, H.MAHFUZ, A. ADNAN, T.LEVENTOURI AND S.ABSAR, “A study of mechanical behavior and morphology of carbon nanotube reinforced UHMWPE/NYLON 6 hybrid polimer nanocomposite fiber", Fibers and polimers, v.15, n.7, pp. 1484-1492, 2014.

[25] KHAN, M.R., MAHFUZ, H., LEVENTOURI, T., et al., "Enhancing toughnes of low-density polyethylene filaments through infusion of multiwalled carbon nanotubes and ultrahigh molecular weight polyethylene", Polimer Engineering and Science, v.51, pp. 654-662, 2011.

[26] CHOI, P., "Molecular dynamics studies of the thermodynamics of HDPE/butane-based LLDPE blends", Polymer, v. 41, pp. 8741-8747, 2000. 